\title{
Participando da esporteterapia: percepção de pais de crianças com paralisia cerebral
}

http://dx.doi.org/10.11606/1807-5509201800020243

\author{
Marina de Brito BRANDÃO* \\ Guilherme Maia Sette CÂMARA** \\ Márcio Cançado Valadares RIBEIRO*** \\ Marisa Cotta MANCINI*
}

*Departamento de Terapia Ocupacional, Universidade Federal de Minas Gerais, Belo Horizonte, MG, Brasil. **Serviço de Esporteterapia Associação Mineira de Reabilitação, Belo Horizonte, MG, Brasil.

\section{Resumo}

0 objetivo do estudo foi compreender a percepção de pais de crianças com paralisia cerebral de grave comprometimento motor sobre a participação de seus filhos nas atividades da esporteterapia na Associação Mineira de Reabilitação (AMR). Foi realizado um estudo qualitativo com doze pais de crianças com paralisia cerebral de grave comprometimento motor. As crianças frequentavam semanalmente as aulas de esporteterapia na AMR. Entrevistas semiestruturadas com os pais das crianças foram realizadas e compreenderam questões relacionadas às suas expectativas acerca do serviço e entendimento das atividades propostas, aspectos positivos e negativos relacionados à participação das crianças nas atividades, possíveis mudanças percebidas pelos pais após a entrada da criança nas aulas de esporteterapia e sugestões de melhorias do serviço. As entrevistas foram transcritas e analisadas por meio da análise de conteúdo. Foram evidenciadas três categorias temáticas: (1) Esporte, reabilitação ou socialização através do brincar?; (2) Facilitadores e barreiras para participação na esporteterapia; (3) Socialização, comportamento e movimento. As famílias apontaram que a participação das crianças no serviço de esporteterapia promoveu beneficios relacionados aos aspectos motores, ao comportamento e à socialização das crianças. A relação entre os instrutores de esporteterapia e as crianças e suas famílias parece ser elemento importante para promoção da participação das crianças nas atividades. Tais informações podem subsidiar a promoção de atividades esportivas em serviços de reabilitação voltados para crianças com deficiência.

Palavras-chave: Esporte adaptado; Crianças com deficiência; Estudo qualitativo; Pais; Reabilitação.

\section{Introdução}

O engajamento de crianças com deficiência em atividades físicas tem por objetivo promover a saúde e a funcionalidade ${ }^{1-5}$, por meio de ganhos motores, emocionais e sociais ${ }^{1,-9-9}$. Especificamente para crianças e jovens com paralisia cerebral (PC), a prática de atividades físicas pode acarretar diversos benefícios, como melhora da força muscular ${ }^{10,11}$, capacidade aeróbica $^{11,12}$ e anaeróbica ${ }^{12,13}$, e possibilidade de experimentaçáo de novos movimentos ${ }^{6,12}$. Com relação aos atributos emocionais e sociais, o envolvimento em atividades físicas pode promover reforço da auto-estima ${ }^{9}$ e melhora da saúde mental ${ }^{12}$, bem como facilitar a socialização do indivíduo com PC nas atividades desempenhadas em grupo ${ }^{11,13-14}$.

A literatura acerca de programas de atividade física para crianças com deficiência centra-se, principalmente, em açóes na escola, por meio da educação física escolar ${ }^{8,15-17}$. Entretanto, CoATes e WiCKERMAN ${ }^{17}$ apontam que crianças com deficiência têm participação restrita na educação física escolar 
devido à falta de provisão de locais, oportunidades e serviços apropriados que atendam às demandas dessa população. Além disso, são poucos os serviços na comunidade que conseguem proporcionar às crianças com deficiência a participação efetiva em atividades esportivas e de lazer ${ }^{18,19}$.

Frente aos benefícios da participação de crianças com deficiência na prática de atividades físicas e à escassez de programas de esporte direcionados especificamente a crianças com deficiência, instituições de reabilitação vem implementado a prática esportiva no processo de reabilitação ${ }^{6,20-21}$. A esporteterapia é uma ação de terapia pelo movimento, que visa desenvolver um comportamento orientado para saúde física, mental e para o bem-estar social, por meio de recursos do esporte ${ }^{21}$. Essa prática pode incluir atividades de recreação, de socialização e de modalidades esportivas, sem o caráter competitivo. As principais características de um programa de esporteterapia envolvem a provisão de atividades adaptadas e graduadas que visem estimular potencialidades motoras (coordenação, equilíbrio, força, percepção espaçotemporal, habilidades motoras remanescentes), emocionais (força de vontade, autoestima, senso de auto-eficácia) e sociais (aumento da interação e socialização) ${ }^{21}$. Espera-se, por meio de programas de esporteterapia, que a criança com deficiência em geral e com PC em específico, sinta-se capaz de engajar-se em

\section{Método}

Foi desenvolvido um estudo qualitativo, utilizando abordagem fenomenológica ${ }^{22}$, com 12 pais (10 mães e 2 pais) de crianças com PC. Esse estudo foi aprovado pelo Comitê de Ética em Pesquisa da Universidade FUMEC (protocolo 02/2011) e os pais assinaram termo de consentimento livre e esclarecido. A seleção da amostra foi proposital, seguindo os seguintes critérios: pais ou responsáveis por crianças com PC com grave comprometimento motor (i.e., classificadas nos níveis IV ou $\mathrm{V}$ do Sistema de Classificação da Função Motora Grossa-GMFCS ${ }^{23}$ que frequentavam as aulas de esporteterapia da AMR. O método de amostragem por saturação foi utilizado para definir o número total de participantes deste estudo. Desta forma, considerou-se a não necessidade de inclusão de novos participantes no estudo quando as informaçóes fornecidas por novos entrevistados passaram a atividades físicas em outros contextos, como na escola ou comunidade. $\mathrm{Na}$ escola, por exemplo, as próprias crianças com deficiência podem auxiliar seus professores e colegas a adaptar as atividades para permitir a participação de todos, uma vez que ela já vivenciou a oportunidade de realizar essas atividades, com sucesso, no ambiente de reabilitação. Dessa forma, programas de esporteterapia podem funcionar como um mecanismo facilitador da inclusão de crianças com deficiência no contexto escolar.

Em reconhecimento aos benefícios do esporte no processo de reabilitação de crianças com deficiência, o programa de esporteterapia da Associação Mineira de Reabilitação (AMR) tem por objetivo promover a reabilitação e estimular o desenvolvimento social e psicomotor de crianças com deficiência física, por meio da prática de atividades físicas e esportivas. Devido à escassez de estudos científicos sobre as açóes e benefícios da esporteterapia no processo de reabilitaçáo de crianças com deficiência, fazse importante conhecer a perspectiva dos pais em relação ao serviço oferecido pela esporteterapia em um centro de reabilitação infantil. Sendo assim, o objetivo do presente estudo foi investigar a percepçáo de pais de crianças com PC de grave comprometimento motor sobre a participação das crianças nas atividades desenvolvidas pelo setor de esporteterapia da AMR.

se repetir com frequência, pouco acrescentando aos dados já coletados ${ }^{24}$. Informaçóes descritivas, como idade, sexo e nível de gravidade motora da criança, bem como parentesco do entrevistado com a criança, estão apresentadas na TABELA 1 . Os nomes das crianças foram substituídos por nomes fictícios para garantir anonimato.

As aulas da esporteterapia ocorrem em grupos de seis a 15 crianças com deficiência física, sendo a maioria crianças com PC, contando ainda com três a quatro instrutores de esporteterapia por aula. Todos os instrutores eram graduados em Educação Física e contavam com a participação de dois estagiários do mesmo curso. O planejamento das aulas é realizado quadrimestralmente para todos os grupos do programa e inclui temas relacionados à saúde, percepção corporal, meio ambiente e cultura. De acordo com cada tema, são utilizados jogos adaptados 
(e.g., basquete, handebol, futebol), modalidades paralímpicas (e.g., atletismo, bocha), brincadeiras adaptadas (e.g., rodas, rouba-bandeira) e/ou atividades rítmicas (e.g., dança, capoeira). As atividades são previamente selecionadas e englobam habilidades motoras (e.g., movimentaçáo livre em tatames e na cadeira de rodas, rolar, correr, saltar), habilidades emocionais (e.g., regulação do comportamento, prazer, diversão) e sociais (e.g., interação com outras crianças e com os professores, respeito a regras). As aulas são direcionadas para todo o grupo, mas os instrutores oferecem assistência e adaptação individualizada, para que crianças com diferentes habilidades e dificuldades possam participar, de forma mais autônoma possível, das atividades propostas para o grupo. Semanalmente, são reportadas as aulas ministradas em um anedotário, visando garantir o seguimento das atividades propostas e observação de possíveis progressos do grupo. Os coordenadores do programa eram responsáveis por realizar as avaliaçóes dos conteúdos ministrados, por meio de consulta aos anedotários e acompanhamento das atividades.

TABELA 1 - Informações descritivas das crianças (idade, sexo e nível de gravidade motora) e parentesco do entrevistado com a criança.

\begin{tabular}{lccccc}
\hline $\begin{array}{l}\text { Nome fictício da } \\
\text { criança }\end{array}$ & Sexo & Idade & GMFCS & $\begin{array}{c}\text { Outras modalidades } \\
\text { de reabilitaçáo }\end{array}$ & $\begin{array}{c}\text { Parentesco do } \\
\text { entrevistado }\end{array}$ \\
\hline Sérgio & Masculino & 5 anos e 8 meses & V & FT, TO, FO & Mãe \\
Aline & Feminino & 10 anos e 9 meses & V & FT, TO, FO & Mãe \\
Bárbara & Feminino & 7 anos e 2 meses & V & FT, TO & Mãe \\
Álvaro & Masculino & 7 anos e 4 meses & IV & FT, TO, FO & Mãe \\
Laura & Feminino & 11 anos e 1 mês & V & FT, FO & Mãe \\
Paulo & Masculino & 11 anos e 7 meses & V & FT, TO, FO & Mãe \\
Lucas & Masculino & 5 anos e 9 meses & V & FT, TO & Mãe \\
Cecília & Feminino & 9 anos e 11 meses & IV & FT, TO & Pai \\
Tales & Masculino & 8 anos e 2 meses & IV & FT, TO & Mãe \\
Tomaz & Masculino & 11 anos e 7 meses & V & FT, TO, FO & Mãe \\
Marcelo & Masculino & 5 anos e 9 meses & V & TO, FO & Mãe \\
Leonardo & Masculino & 8 anos e 5 meses & V & FT, TO, FO & Pai \\
\hline
\end{tabular}

GMFCS: Sistema de Classificação da Função Motora Grossa $^{23}$. Os níveis IV e $V$ do GMFCS indicam grave comprometimento motor, com necessidade de uso de cadeira de rodas e apoio para manutenção da postura sentada. Outras modalidades de reabilitação (1 a 2 sessões semanais de 45 minutos): FT- Fisioterapia, TO Terapia Ocupacional FO- Fonoaudiologia.
As aulas são constituídas de três momentos, sendo iniciada com a recepção aos alunos. Os objetivos desse primeiro momento são receber os alunos em um ambiente alegre e receptivo, contextualizar o tema determinado no planejamento e preparar os alunos para o trabalho principal. O segundo momento é constituído pelo trabalho dos tópicos previamente especificados no planejamento, como habilidades motoras, perceptuais e sociais, por meio de atividades lúdicas e esportivas. Por fim, o processo se encerra com o momento de relaxamento, que visa a diminuição da frequência cardíaca e também do estado de excitação da criança. As aulas ocorrem uma vez por semana, com duração de 1 hora. Todas as crianças cujos pais participaram do estudo tinham frequência semanal na esporteterapia por pelo menos
6 meses anteriores ao início do estudo. Além disso, as crianças eram atendidas semanalmente nos serviços de fisioterapia, fonoaudiologia e terapia ocupacional. As modalidades de atendimento a cada criança do estudo estão apresentadas na TABELA 1.

A coleta de dados ocorreu através de entrevistas semiestruturadas com os pais das crianças. A entrevista compreende um grupo de perguntas cujo objetivo é guiar o entrevistado acerca das informaçôes requeridas pelo pesquisador ${ }^{25}$. Para o presente estudo, elaborou-se um roteiro de entrevista com os seguintes pontos norteadores: 1) forma de ingresso no serviço, 2) expectativas dos pais sobre o programa de esporteterapia, 3) condução das aulas, 4) percepçáo sobre os pontos positivos e negativos das aulas, 5) mudanças observadas nas 
crianças participantes do programa, 6) representação da esporteterapia para suas crianças. As entrevistas realizadas tiveram duração média de 20 minutos. Elas foram gravadas com gravador digital Sony icd-bx800 e, posteriormente, transcritas a fim de se realizar uma análise cuidadosa dos dados. As entrevistas foram realizadas ao longo de 2 meses, por estagiária de terapia ocupacional previamente familiarizada com procedimentos do método qualitativo, que não tinha contato prévio com as famílias ou com o programa de esporteterapia da instituição.

As entrevistas foram analisadas por meio da análise de conteúdo, processo de prática interpretativa cujo objetivo é atingir uma significação profunda do texto $^{26}$. Tal análise é composta por diversas etapas nas quais primeiramente realiza-se uma pré-análise do material, utilizando-se de leitura flutuante e posterior agrupamento dos indicadores levantados em unidades de análise. Por fim, é efetuado o tratamento e a interpretaçáo dos dados das categorias temáticas ${ }^{27}$. A análise de conteúdo foi feita pela entrevistadora e por uma terapeuta ocupacional. A estagiária não tinha conhecimento sobre o programa de esporteterapia e sobre o teor da pesquisa. Para garantir que a saturação da amostra, após cada entrevista, realizava-se a transcrição e leitura flutuante do material pelas duas examinadoras. Quando observou-se que os conteúdos apresentaram repetiçôes, e novas informaçóes não estavam sendo reveladas, sob a perspectiva das duas examinadoras, optou-se por encerramento das entrevistas. A partir desse momento, foram estabelecidas as codificaçóes dos dados e estabelecimento das categorias temáticas, de comum acordo entre as examinadoras.

\section{Resultados e Discussão}

As categorias temáticas levantadas a partir das entrevistas com os pais foram: (1)"Esporte, reabilitação ou socialização através do brincar?", (2) "Facilitadores e barreiras para participação na esporteterapia" e (3)
"Socialização, comportamento e movimento". A última categoria foi subdividida em: (a) "Conhecendo limites e potencialidades" e (b) "Ele tem vontade de brincar". A Figura 1 ilustra a organização das categorias temáticas.

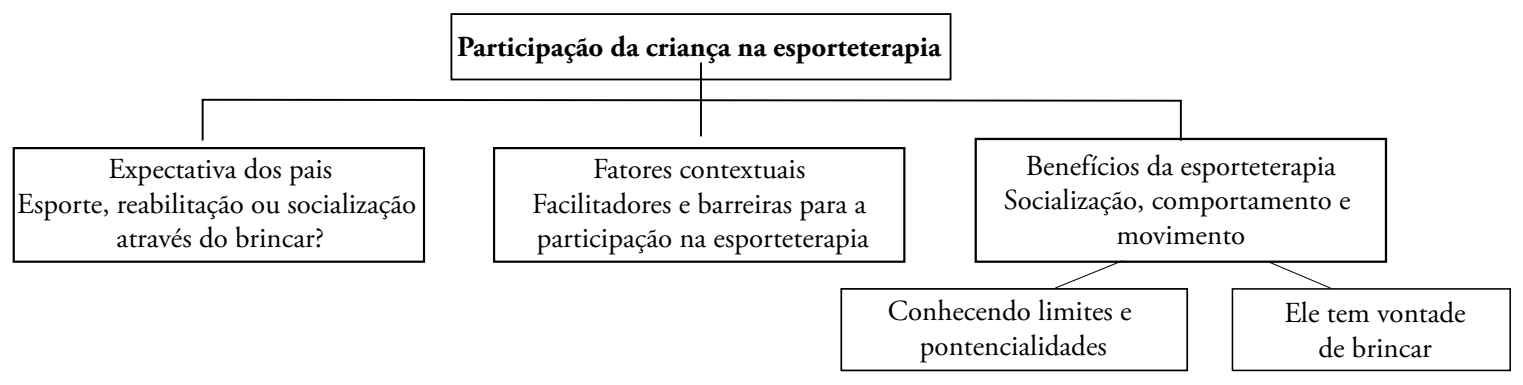

FIGURA 1 - Compreensão da participação de crianças com PC em programa de esporteterapia.

\section{Esporte, reabilitaçáo ou socializaçáo através do brincar?}

Houve uma variedade de relatos dos pais acerca de suas expectativas no início do trabalho da esporteterapia, que incluíram a perspectiva da atividade enquanto prática de modalidades esportivas, reabilitação física ou possibilidade de socialização pelo brincar.

Enquanto alguns pais reportaram a expectativa de realizaçáo de modalidades esportivas durante a esporteterapia "Eu achei que era esporte, igual esporte normal. Tipo jogar bola” (mãe da Laura), outros apontaram a dificuldade inicial de entendimento de como seria possível a participação da criança no esporte devido à grave limitação motora " $M e$ falaram que era esporteterapia né, e esporte em si ela não consegue né, ela não consegue mesmo fazer" (mãe da Bárbara). A maioria dos pais esperava, inicialmente, que fossem realizadas atividades físicas de reabilitação "Eu achei que era tipo a fisioterapia, que ela ia fazer exercícios. Achei que era uma atividade física mais pesada" (pai da Cecília), "Eu achei que era só exercício físico mesmo, exercício o tempo todo" (mãe do Tomaz). A expectativa de dois pais estava relacionada à oportunidade de socialização de seus 
filhos com outras crianças e com os terapeutas da instituição, por meio do brincar. " $E$ isso mesmo que eles fazem, de brincar de bola, e colocar ele pra brincar com outras crianças também" (mãe do Sérgio); "É, eu achava que era brincadeira, como tem mesmo, mas eu também achava que era uma oportunidade dele conviver de perto com os profissionais que cuidam dele" (pai do Leonardo).

Apesar do desconhecimento inicial de alguns pais, ao longo do trabalho realizado pela esporteterapia, os mesmos demonstraram compreensão das características do serviço, por meio da participação nas atividades "Porque tinham algumas mães que estavam achando que era só brincar. Ai chamaram a gente para fazer, jogar bola, e eu cansei muito. Na metade do horário eu já estava cansada. E é aí que a gente vê que não é brincadeira não" (mãe da Laura). Assim, é possível que a vivência dos pais nas atividades da esporteterapia possibilite o entendimento do trabalho proposto.

A expectativa dos pais acerca da esporteterapia enquanto reabilitação física, similar aos atendimentos convencionais de reabilitação, revela que programas de recreação e esporte para crianças com PC ainda são uma área de conhecimento limitada ${ }^{9}$. Frente ao desconhecimento do trabalho a ser realizado, os pais, frequentando um centro de reabilitação infantil, esperavam que fossem realizadas atividades semelhantes às modalidades de intervenção já conhecidas, como a fisioterapia. A dificuldade de entendimento dos pais acerca da esporteterapia com crianças com deficiência pode estar relacionada também às limitaçóes funcionais das crianças. Palisano et al. ${ }^{28}$ apontam que crianças com PC de grave comprometimento motor apresentam maior restrição na participação de atividades recreacionais e de lazer em relação às crianças com gravidade motora leve. Entretanto, os autores apontam que a participação das crianças com PC nessas atividades decorre náo somente de fatores relacionados às alteraçóes do sistema neuromusculoesquelético, mas também de fatores contextuais como atitude de pais e professores ${ }^{28}$. Tsai e FUNG ${ }^{18}$, em um estudo qualitativo com pais de crianças com deficiência mental acerca da participação de seus filhos em um programa de participação esportiva inclusiva, apontaram que os pais náo acreditavam que seus filhos pudessem se engajar em atividades do esporte, apresentando dificuldades em compreender, incialmente, os possíveis benefícios da educação física para seus filhos, característica também evidenciada no presente estudo.

\section{Facilitadores e barreiras para participaçáo na esporteterapia}

Cinco pais reportaram que seus filhos apresentaram dificuldades iniciais para participar das atividades da esporteterapia, devido às especificidades da aula, como presença de várias crianças e adultos, realização de atividades em grupo, músicas, interaçôes verbais frequentes entre crianças e instrutores. Os pais reportaram que, ao longo do tempo, as crianças apresentaram crescente tolerância aos estímulos sonoros e demonstraram maior envolvimento nas atividades. "Ele vinha chorando, vinha berrando mesmo. Porque ele não gosta de barulho né, e lá tem muito barulho, tem música. Ai ele ficava irritado. Mas hoje ele gosta, ele adora" (mãe do Álvaro). "Antes a gente começava a brincar e ela brincava um pouco e cansava. Agora ela já suporta mais tempo" (mãe da Aline).

Um elemento que parece ter contribuído para maior envolvimento das crianças na esporteterapia, segundo a maioria dos pais, refere-se à relação entre instrutores, crianças e suas famílias. O ambiente acolhedor criado pelos instrutores facilitou o envolvimento das crianças nas atividades. Algumas características dos instrutores, como empenho "les são muito empenhados, empenhados mesmo"(mãe da Aline) e relacionamento positivo com os pais "A felicidade, o carinho que eles tratam a gente. Porque eu sei que qualquer coisa que en preciso eu posso ir lá falar com eles" (máe do Paulo) foram reportadas como importantes para o envolvimento das crianças e das famílias na esporteterapia.

Por outro lado, alguns pais apontaram receios relacionados aos cuidados com a criança durante as aulas da esporteterapia. Dois pais relataram insatisfação com relação à retirada de aparelhos ortopédicos e com a aparência da criança após as aulas "ruim é só que eles tiram o tutor da criança e não colocam. Devolvem sem o tutor, descabelados" (mãe da Laura), além de um pai que reportou a importância da provisão de cadeira de rodas individualizada para a criança durante as aulas "Porque eles tem várias cadeiras lá, e a Cecilia é muito magrinha. Ai, se ela fica em uma cadeira de rodas grande demais, ela fica insegura. Uma cadeira própria pra ela dá mais segurança e os pais ficam mais tranquilos também" (pai da Cecília). Com relação à segurança, foi reportado ainda o receio de três pais pela não assistência individualizada à criança "Acho que são poucos profissionais para muita criança. É muita gente mesmo, então a gente fica até com receio. Pela segurança das crianças mesmo" (mãe do Álvaro).

Os resultados do presente estudo apontam que um dos principais facilitadores da participação das 
crianças no programa de esporteterapia foi a atitude positiva dos instrutores. Segundo Tsai e FUnG ${ }^{18}$, os instrutores de atividades esportivas e programas de recreação devem oferecer suporte à participação de pessoas com deficiência em atividades físicas por meio da acomodação das necessidades e habilidades do indivíduo com a atividade proposta. Para tanto, os profissionais devem demonstrar habilidades para lidar com as crianças e famílias e conhecer suas habilidades e dificuldades ${ }^{1}$.

Com relação às barreiras, a participação de crianças com PC em atividades esportivas pode ser restrita devido ao medo dos pais das crianças se machucarem ${ }^{9}$, pela percepção das limitaçóes funcionais da criança ${ }^{1,9}$ e pelas dificuldades de compreensão das atividades mais apropriadas para as habilidades e interesses da criança ${ }^{1}$. Sendo assim, é necessário o estabelecimento de comunicação efetiva dos instrutores de educação física com os pais, de forma a criar uma relaçáo de confiança ${ }^{19}$.

\section{Socializaçáo, comportamento e movimento}

\section{Conbecendo limites e potencialidades}

Um aspecto positivo atribuído pelos pais ao trabalho realizado na esporteterapia refere-se à oportunidade de socialização da criança, reportada por todos os pais " $E$ o convivio com outras crianças. E com adultos também" (pai do Leonardo); "Então eu acho que ajudou foi isso, dela fazer mais amizades. Ela conheceu um tanto de gente lá na esporteterapia, então foi muito bom" (pai da Cecília). A importância atribuída à promoção da socializaçáo durante as aulas da esporteterapia parece estar relacionada à restriçáo de oportunidades de interaçáo social da criança nas outras modalidades de atendimento do centro de reabilitação, segundo relato de duas mães "E essa oportunidade de estar com outras crianças com problemas diferentes dela, de tá socializando. A socialização entre eles mesmos, porque com o atendimento separado eles acabam nem reparando entre si. E aqui não, é todo mundo misturado. Idades diferentes, problemas diferentes" (mãe da Aline) e na comunidade "Acho que éa interação com outras crianças. Que talvez, em outros lugares, o Álvaro não poderia ter essa interação direito né" (mãe do Álvaro). Além disso, a oportunidade de socializaçáo com outras crianças e adultos durante as aulas de esporteterapia foi entendida por um pai como uma preparação para a inserção da criança na escola "Pra ele tá sendo bom porque tá sendo como uma preparação pra ele ir pra escola. Então conviver com outros adultos tá sendo bom"(pai do Leonardo).
Os pais apresentaram opinióes diversas acerca da presença de crianças de diferentes idades e gravidade de comprometimento motor na mesma aula de esporteterapia. Alguns pais apontaram que a diversidade do convívio pode trazer benefícios para o entendimento da criança acerca de suas habilidades e limitaçōes "Por exemplo, no dia em que teve brincadeira de colorir, pra Aline não foi legal porque a coordenação dela não é boa. Mas por outro lado, tem brincadeiras que ela consegue e o outro náo consegue. Eu acho que é bom de ela ver que tem coisas que ela não consegue, mas que o coleguinha dela consegue. Que às vezes a gente que é mãe não consegue passar, porque a gente acaba fazendo por eles" (mãe da Aline). Por outro lado, tal diversidade foi vista pela maioria dos pais como elemento dificultador para a participaçáo da criança “(...) de uns tempos pra cá eu venho achando que ele tá meio sem paciência. É que o Paulo é muito inteligente né, entäo eu acho que ele acha algumas brincadeiras meio bobinhas, ele meio que perde a paciência. (...) Porque eu acho que esse éo problema de colocar todo mundo misturado. Gente de idade diferente e de capacidade diferente também" (mãe do Paulo).

Uma das principais açóes da esporteterapia é promover a socialização das crianças, em um ambiente lúdico, desafiador e adaptado ${ }^{21}$. Samalot-Rivera e Porretta ${ }^{16}$ observaram a importância da oportunidade de socializaçáo de crianças com deficiência nas aulas de educaçáo física. Os autores investigaram a percepção de professores de educação física adaptada no aprendizado de habilidades sociais, que salientaram a importância de ensinar aos alunos com deficiência, habilidades sociais como interação social, lidar com outras pessoas e situaçóes, fazer e manter amigos e resolver problemas ${ }^{16}$. A maioria dos pais, entretanto, apontou que um dos elementos negativos do programa de esporteterapia estava relacionado à heterogeneidade de comprometimento motor das crianças. Sendo assim, o programa de esporteterapia depara-se com o grande desafio de oferecer serviço que atenda às demandas específicas de cada criança e que, ao mesmo tempo, proporcione ambiente inclusivo com vivências de interação social, solução de problemas e expressão funcional.

\section{Ele tem vontade de brincar}

Algumas mudanças no comportamento da criança decorrentes do trabalho da esporteterapia foram apontadas pelos pais, tais como maior interesse $\mathrm{da}$ criança pelo brincar, manifestado por mudança do humor apontada por seis pais "O Paulo tá mais alegre né. OPaulosempresaimais alegre de lá. (...) "(mãe do Paulo), 
aumento do nível de alerta e paciência, reportada por duas mães "Ele ficou melhor pra brincar. Ficou mais disposto e com mais paciência também. (...) Ah melhorou né, melhorou tudo isso aî" (mãe do Sérgio), e, segundo uma mãe, da auto-estima "É a auto estima né, que ela dá pro Lucas, e para as mães também. Você viu o uniforme deles agora que coisa mais linda. $O$ Lucas se sente super importante com aquela blusa" (mãe do Lucas). Essas mudanças parecem ultrapassar o ambiente do centro de reabilitação e transferir-se para outros ambientes, de acordo com o relato de duas mães, como no ambiente domiciliar "Agora ela já tem mais vontade de brincar com a gente em casa" (mãe da Aline) e comunitário "Na rua as pessoas perguntam e eu digo que ele tá indo praticar esporte. Ele fica todo feliz" (mãe do Lucas).

Três pais também reportaram aumento da movimentação espontânea da criança no brincar, após início do trabalho da esporteterapia "Eu acho que ela mexe mais com as mãos, ela tem mais vontade de pegar as coisas. Eu acho que foi bom pra ela. Ela até joga as coisas pra cima, no chão. Então eu acho que foi muito bom" (mãe da Laura). As mudanças observadas pelos pais nas habilidades de socializaçáo, comportamento e movimentação da criança atribuídas ao trabalho da esporteterapia ilustram a importância do esporte para pessoas com deficiência e corroboram com a literatura ${ }^{1-3,9,11,13}$.

O presente estudo apresenta limitaçóes. Os pais das crianças reportaram benefícios relacionados à socialização, aumento da movimentaçáo espontânea e regulação de comportamento. Não se sabe, entretanto, se tais características estão relacionadas exclusivamente ao programa de esporteterapia ou da associação com modalidade de reabilitação concomitante, uma vez que todas as crianças recebiam outras modalidades de intervenção (fisioterapia, fonoaudiologia, terapia ocupacional). Tal característica deve-se às necessidades das crianças com $\mathrm{PC}$ de grave comprometimento motor de atendimentos multidisciplinares. Além disso, esse estudo avaliou a percepção dos pais de um único programa de esporteterapia, que acontece em uma instituição de reabilitação. Assim, não é possível generalizar as informaçôes do estudo para todas as atividades e programas de esporteterapia, principalmente para aqueles com caraterísticas diferentes. Futuros poderão analisar a eficácia dessa modalidade de intervenção na socialização, movimentação e regulação de comportamento de crianças com PC, de diferentes programas de esporteterapia, por meio de estudos quantitativos experimentais.

O presente estudo visou compreender a percepção dos pais de crianças com PC de grave comprometimento motor acerca de um programa de esporteterapia em um centro de reabilitação infantil. Os pais apontaram que os benefícios do programa estão centrados, principalmente, na melhoria das habilidades sociais das crianças e que a relação entre os instrutores e as crianças e suas famílias parece ser elemento fundamental para o aumento da participação das crianças nas atividades propostas.

\section{Abstract}

\section{Participating in a sport therapy program: perception of parents of children with cerebral palsy}

The aim of the study was to understand the perception of parents of children with cerebral palsy with severe motor impairments, regarding children's participation in activities at an adapted sport program at Associação Mineira de Reabilitação (AMR). A qualitative study was conducted with twelve caregivers of children with cerebral palsy with severe motor impairments. The children took part, weekly, on the adapted sport program at AMR. Semi-structured interviews with children's caregivers were performed and they encompassed questions related to caregivers' expectations regarding the services and the understanding about the proposed activities, positive and negative aspects related to children's participation, observed possible children's changes after the beginning of the sport program and suggestions to improve services. The interviews were transcribed and analyzed through content analysis. Three thematic categories were found: (1) Sport, rehabilitation or socialization through play?;(2) Facilitators and barriers of participation at the sport program; (3) Socialization, behavior and movement. Families reported that the children's participation in the adapted sport program led to improvements on children's motor, behavior and social skills. The relationship between instructors and children and their families seem to be an important element 
to promote children's participation in the proposed activities. Such information can subside the use of sport activities in rehabilitation services for children with disabilities.

KeYwords: Adapted sport; Children with disabilities; Qualitative study; Caregivers; Rehabilitation.

\section{Referências}

1. Verschuren O, Wiart L, Hermans D, Ketelaar M. Identification of facilitators and barriers to physical activity in children and adolescents with cerebral palsy. J Pediatr. 2012;161:488-94.

2. Zwier JN, Van Schie PE, Becher JG, Smits DW, Gorter JW, Dallmeijer AJ. Physical activity in young children with cerebral palsy. Disabil Rehabil. 2010;32:1501-8.

3. Carroll KL, Leiser J, Paisley TS. Cerebral palsy: physical activity and sport. Cur Sports Med Rep. 2006;5:319-22.

4. Chawla JC. Sports for people with disability. BMJ.1994;308:1500-4.

5. Strapasson AM, Duarte E. Proposta de ensino polybat para pessoas com paralisia cerebral. Arq Ciênc Saúde UNIPAR. 2006; 10:165-75.

6. Labrocini RHDD, Cunha MCB, Oliveira ASB, Gabbai AA. Esporte como fator de integração do deficiente físico na sociedade. Arq Neuropsiq. 2000;58:1092-9.

7. Maiano C, Ninot G, Morin AJS, Bilard J. Effects of sport participation on the basketball skills and physical self of adolescents with conduct disorders. Adapt Phys Activ Q. 2007;24:178-96.

8. Hein V, Hagger MS. Global self-steem, goal achievement orientations, and self-determined behavioral regulations in a physical education setting. J Sports Sci. 2007;25:149-59.

9. Wind WM, Schwend RM, Larson J. Sports for physically challenged child. J Am Acad Orthop Surg. 2004;12:126-37.

10. Peungsuwan P, Parasin P, Siritaratiwat W, Prasertnu Y, Yamauchi J. Combined exercise training on functional performance in children with cerebral palsy: a randomized-controlled trial. Pediatr Phys Ther. 2017;29:39-46.

11. Verschuren O, Ketelaar M, Gorter JW, Helders PJ, Uiterwaal CS, Takken T. Exercise training program in children and adolescents with cerebral palsy: a randomized controlled trial. Arch Pediatr Adolesc Med. 2007;161:1075-81.

12. Unnithan VB, Katsimanis G, Evangelinou C, Kosmas C, Kandrali I, Kellis E. Effect of strength and aerobic training in children with cerebral palsy. Med Sci Sports Exerc. 2007; 39:1902-9.

13. Slaman J, Van Den Berg-Emons HJ, Van Meeteren J, Twisk J, Van Markus F, Stam HJ, et al. A lifestyle intervention improves fatigue, mental health and social support among adolescents and young adults with cerebral palsy: focus on mediating effects. Clin Rehabil. 2015;29:717-27.

14. Van Wely L, Balemans AC, Becher JG, Dallmeijer AJ. The effectiveness of a physical activity stimulation programme for children with cerebral palsy on social participation, self-perception and quality of life: a randomized controlled trial. Clin Rehabil. 2014;28:972-82.

15. Block M, Obrusnikova I. Inclusion in physical education: a review of the literature from 1995-2005. Adapt Phys Activ Q. 2007;24:103-24.

16. Samalot-Rivera A, Porretta DL. Perceptions and practices of adapted physical educators on the teaching of social skills. Adapt Phys Activ Q. 2009;26:172-86.

17. Coates J, Wickerman P. Empowering children with special educational needs to speak up: experiences of inclusive physical education. Dis Rehab. 2010;32:1517-26.

18. Tsai $\mathrm{EH}$, Fung L. Parents experiences and decisions on inclusive sport participation of their children with intelectual disabilities. Adapt Phys Activ Q. 2009;26:151-71.

19. Columna L, Pyfer J, Senne T, Velez L, Bridenthrall N, Canabal MY. Parental expectations of adapted physical educators: a Hispanic perspective. Adapt Phys Activ Q. 2008;25:228-46.

20. Palisano RJ. Physical activities of children with cerebral palsy: what are the considerations? Dev Med Child Neurol. 2012;54:390-1.

21. Souza PA. Esporteterapia como indutora da neuroplasticidade na paralisia cerebral. In: Lima CLA, Fonseca LF. Paralisia cerebral: neurologia, ortopedia e reabilitaçâo. Rio de Janeiro: Guanabara Koogan; 2004. p. 421-30.

22. Wilding C, Whiteford G. Phenomenological research: an exploration of conceptual, theoretical, and practical issues. OTJR. 2005;25:98-104. 
23. Palisano R, Rosenbaum P, Walter S, Russell D, Wood E, Galuppi B. Development and reliability of a system to classify gross motor function in children with cerebral palsy. Dev Med Child Neurol. 1997;39:214-23.

24. Fontanella BJB, Ricas J, Turato ER. Amostragem por saturação em pesquisas qualitativas em saúde: contribuiçôes teóricas. Cad Saúde Pública. 2008;24:17-27.

25. Haguette TMF. Metodologias qualitativas na sociologia. Petrópolis: Vozes; 2000.

26. Rocha D, Deusdara B. Análise de conteúdo e análise do discurso: aproximaçóes e afastamentos na (re)construção de uma trajetória. ALEA. 2005;7:305-22.

27. Bardin LB. Análise de conteúdo. São Paulo: Martins Fontes; 1997.

28. Palisano RJ, Chiarello LA, Orlin M, Oeffinger D, Polansky M, Maggs J, et al. Determinants of intensity of participation in leisure and recreational activities by children with cerebral palsy. Dev Med Child Neurol. 2011;53:142-9.

\section{Agradecimentos}

Agradecemos aos pais participantes do estudo, aos instrutores do programa de esporteterapia da AMR e à Livia Álvares, que nos auxiliou no processo de coleta e análise dos dados.

\begin{tabular}{r|l} 
ENDEREÇo & \\
Marina Brandão & Recebido para publicação: 28/07/2015 \\
Av. Antônio Carlos, 6627 & Revisado: 23/01/2017 \\
31270-901 - Belo Horizonte-MG - BRASIL & Aceito: 03/o8/2017 \\
e-mail: marinabbrandao@gmail.com &
\end{tabular}


